PROCEEDINGS OF THE

AMERICAN MATHEMATICAL SOCIETY

Volume 137, Number 10, October 2009, Pages 3297-3307

S 0002-9939(09)09360-5

Article electronically published on May 13, 2009

\title{
A FUNCTIONAL-ANALYTICAL APPROACH TO THE ASYMPTOTICS OF RECURSIONS
}

\author{
CHRISTIAN PÖTZSCHE
}

(Communicated by Andreas Seeger)

\begin{abstract}
We propose a functional-analytical method to investigate the longterm behavior of recursions (difference equations). It is based on a formulation of given (implicit) recursions as abstract operator equations in sequence spaces. Solving them using appropriate tools from nonlinear analysis yields quantitative convergence results and equips us with a method to verify summable or subexponential decay.
\end{abstract}

\section{INTRODUCTION}

The classical and generally applicable tools in the stability theory of discrete dynamical systems (or in synonymic terminology, recursions or difference equations) are typically based on estimates using various Gronwall-type inequalities or Lyapunov functions (cf., e.g., Aga00, Chapter 5]). In particular in the autonomous (time-invariant) case, where the law of evolution given by the right-hand side does not depend on time, asymptotic stability of solutions generically goes hand in hand with exponential decay. It is not surprising that one encounters more complex behavior in the general setting of nonautonomous (time-variant) recursions. Here, subexponential decay can occur in the sense that solutions are, for example, only summable. Accordingly, in order to deal with such problems, more flexible convergence notions have been developed using weighted norms (e.g., [Pin98]) or criteria for $\ell^{p}$-summability of solutions (see Gor71] for a method using Lyapunov functions).

In this paper we suggest another approach to stability or attractivity problems for difference equations. It is based on an abstract formulation of a recursion (say in $\mathbb{K}^{d}$ ) incorporating initial conditions, as an operator equation in the infinite-dimensional space $\ell\left(\mathbb{K}^{d}\right)$ of all sequences in $\mathbb{K}^{d}$. The corresponding operator is composed of a trivial embedding map to include initial conditions, as well as a right shift and a substitution (Nemytskii) operator. Choosing an appropriate normed subspace of $\ell\left(\mathbb{K}^{d}\right)$ to capture the specific kind of decay, and appropriate techniques from nonlinear analysis to solve such problems, endows us with quantitative convergence results.

Received by the editors June 20, 2007, and, in revised form, August 27, 2007.

2000 Mathematics Subject Classification. Primary 39A11; Secondary 46T20, 47H09, 47H10, 47J05, 65Q05.

Key words and phrases. Recursion, iteration, nonautonomous difference equation, attractivity, $\ell^{p}$-stability, admissibility, sequence space, functional-analytical method, measure of noncompactness.

(C)2009 American Mathematical Society 3297

Reverts to public domain 28 years from publication 
The advantage of such a reformulation is that proofs become conceptually clear and transparent. Indeed, one only has to show that certain mappings on sequence spaces are well-defined and satisfy a structural assumption guaranteeing that e.g. fixed point theorems can be employed, such as being contractive, completely continuous or condensing. Results of this kind appear to be well-documented in the literature (cf. [AZ90, DG05] or for the linear case [Mad70, Wil84]). Moreover, the proofs follow the same lines for various convergence notions, since only the space setting needs to be modified. Beyond convergence, one also obtains the existence of solutions for implicit problems.

Although the above idea is contiguous, so far it seems rarely analyzed in the literature. The only related reference we are aware of is the work of Siafarikas and his collaborators (see, for example, [PS05]), which is essentially restricted to $\ell^{2}$-convergence. The more flexible situation of arbitrary sequence spaces has been considered in EP07, a paper which focuses on fixed point results (of Banach, Krasnoselski-, Reinermann- and Goebel-Kirk-type) to solve the resulting sequential operator equations.

However, fixed point methods have the disadvantage that the domain and range of the mappings under consideration need to coincide. The paper at hand circumvents this problem by using a result (Theorem 2.1) which easily follows from Darbo's or Sadovskiî's fixed point theorem (cf. Dar55 or Sad67, respectively). Having this tool available we apply our methodology to implicit recursions of the form

$$
x_{k+1}=f_{k}\left(x_{k}, x_{k+1}\right)+g_{k}\left(x_{k}, x_{k+1}\right),
$$

where the mapping $f_{k}$ is assumed to yield an adequate admissibility property. This means that for each perturbation sequence $\psi=\left(\psi_{k}\right)$ from a sequence space $Y$ there exists a unique solution $\phi=\left(\phi_{k}\right)$ for the initial value problem

$$
x_{k+1}=f_{k}\left(x_{k}, x_{k+1}\right)+\psi_{k+1}, \quad x_{0}=\psi_{0}
$$

in a sequence space $X$. Such admissibility results under dichotomy assumptions, among others, can be found in CS67, NP97, Pin98, Sas06. Then, in order to obtain convergence results for (1.1), it will be sufficient to show that the substitution operator induced by $g_{k}$ will have a specific compactness property between $X$ and $Y$. Examples of admissible spaces for semi-linear recursions close our considerations.

\section{Preliminaries and a Darbo-type Result}

We define the discrete interval $\mathbb{N}_{0}:=\{k \in \mathbb{Z}: k \geq 0\}$ of nonnegative integers. As usual, $\mathbb{K}$ denotes the field of real or complex numbers and $\mathbb{K}^{d}$ the $d$-dimensional Euclidean (or Hermitian) space, equipped with norm $|\cdot|$.

Let $X$ be a Banach space with norm $\|\cdot\|_{X}$ and corresponding family $\mathcal{B}(X)$ of bounded subsets. The closed ball in $X$ with radius $\rho$ centered at 0 is denoted by $\bar{B}_{\rho}(X)$. We briefly write $\ell\left(\mathbb{K}^{d}\right)$ for the linear space of sequences $\phi=\left(\phi_{k}\right)_{k \geq 0}$ in $\mathbb{K}^{d}$.

The Hausdorff measure of noncompactness $\chi_{X}: \mathcal{B}(X) \rightarrow[0, \infty)$ is defined as

$$
\chi_{X}(B):=\inf \{\epsilon>0: B \text { has a finite } \epsilon \text {-net in } X\}
$$

(cf. ADL97, pp. 20ff], which will be our standard reference concerning $\chi_{X}$ ). Since it is possible to determine $\chi_{X}$ explicitly for certain spaces $X$ (see below), we will use this particular measure of noncompactness exclusively throughout the paper. 
Well-known properties of $\chi_{X}$ needed here include translation invariance $\chi_{X}(x+B)=$ $\chi_{X}(B)$ for every $B \in \mathcal{B}(X)$ and $x \in X$. Let $\chi_{Y}$ denote the Hausdorff measure of noncompactness on a further Banach space $Y$. Then a mapping $\mathcal{T}: X \rightarrow Y$ is called condensing (see, e.g., ADL97, p. 38, Definition 5.1(b)]) if it is continuous and one has

$$
\chi_{X}(B)>0 \Rightarrow \chi_{Y}(\mathcal{T}(B))<\chi_{X}(B) \quad \text { for all } B \in \mathcal{B}(X) .
$$

In concrete applications it is frequently difficult to verify the above implication, and one needs sufficient conditions for (2.1) to hold. For example, a continuous mapping $\mathcal{T}$ is condensing if it is completely continuous (one has $\chi_{Y}(\mathcal{T}(B))=0$ for $B \in \mathcal{B}(X))$, or a set-contraction, where we have a $\lambda \in[0,1)$ such that

$$
\chi_{Y}(\mathcal{T}(B)) \leq \lambda \chi_{X}(B) \text { for all } B \in \mathcal{B}(X) .
$$

Also contractive mappings are set-contractions and more general, if $\mathcal{T}: X \rightarrow Y$ satisfies a global Lipschitz condition with constant lip $\mathcal{T}$, then it follows that

$$
\chi_{Y}(\mathcal{T}(B)) \leq \operatorname{lip} \mathcal{T} \chi_{X}(B) \text { for all } B \in \mathcal{B}(X) .
$$

After these preparations we arrive at

Theorem 2.1. Let $\mathcal{A}: D(\mathcal{A}) \subseteq X \rightarrow Y$ and $\mathcal{G}: X \rightarrow Y$ be mappings satisfying:

(i) there exist reals $\alpha, \beta, \gamma \geq 0$ such that $\|\mathcal{G}(x)\|_{Y} \leq \alpha+\beta\|x\|_{X}^{\gamma}$ for all $x \in X$,

(ii) $\mathcal{A}^{-1}: Y \rightarrow X$ exists and is globally Lipschitz with $\mathcal{A}^{-1}(0)=0$,

(iii) $\mathcal{A}^{-1} \circ \mathcal{G}: X \rightarrow X$ is a set-contraction,

(iv) the inequality $\left(\alpha+\beta \rho^{\gamma}\right) \operatorname{lip} \mathcal{A}^{-1} \leq \rho$ admits a solution $\rho_{0}>0$.

Then the set $\{x \in X: \mathcal{A}(x)=\mathcal{G}(x)\} \subseteq \bar{B}_{\rho_{0}}(X)$ is nonempty and compact.

Remark 2.2. (1) Instead of being a set-contraction, in order to ensure that there exists a solution of the coincidence equation $\mathcal{A}(x)=\mathcal{G}(x)$ in $\bar{B}_{\rho_{0}}(X)$, one can assume that the composition $\mathcal{A}^{-1} \circ \mathcal{G}: X \rightarrow X$ is condensing. For this generalization, Sadovski1's theorem (as stated in [ADL97, p. 40, Theorem 5.4]) has to be used in the subsequent proof.

(2) It is elementary to derive criteria sufficient for hypothesis (iv). Examples are given in the following three cases:

- $\gamma<1$,

- $\gamma=1$ and $\beta \operatorname{lip} \mathcal{A}^{-1}<1$,

- $\gamma>1, \beta>0$ and $(\beta \gamma)^{\frac{1}{1-\gamma}}\left[\alpha+\beta(\beta \gamma)^{\frac{\gamma}{1-\gamma}}\right] \operatorname{lip} \mathcal{A}^{-1} \leq 1$.

Proof of Theorem 2.1, By assumption the composition $\mathcal{A}^{-1} \circ \mathcal{G}: X \rightarrow X$ satisfies

$$
\left\|\mathcal{A}^{-1}(\mathcal{G}(x))\right\|_{X} \leq \operatorname{lip} \mathcal{A}^{-1}\|\mathcal{G}(x)\|_{Y} \leq \operatorname{lip} \mathcal{A}^{-1}\left(\alpha+\beta \rho_{0}^{\gamma}\right) \leq \rho_{0} \quad \text { for all } x \in \bar{B}_{\rho_{0}}(X)
$$

and therefore $\mathcal{A}^{-1} \circ \mathcal{G}$ maps the bounded, closed and convex set $\bar{B}_{\rho_{0}}(X)$ into itself. Thus, thanks to assumption (iii), we can apply Darbo's theorem (cf. BG80, p. 17, Theorem 5.1]) to verify that the set of fixed points for $\mathcal{A}^{-1} \circ \mathcal{G}$ is nonempty compact. Obviously, solutions of $\mathcal{A}(x)=\mathcal{G}(x)$ are fixed points of $\mathcal{A}^{-1} \circ \mathcal{G}$ and vice versa. 
Our goal is to deduce attractivity properties for forward solutions of (1.1) from Theorem 2.1. Thereto, the following complete normed subspaces of $\ell\left(\mathbb{K}^{d}\right)$ are crucial:

$$
\begin{array}{rlrl}
\ell^{\infty}\left(\mathbb{K}^{d}\right):=\left\{\phi=\left(\phi_{k}\right)_{k \geq 0}: \sup _{k \geq 0}\left|\phi_{k}\right|<\infty\right\}, & & \|\phi\|_{\ell^{\infty}}:=\sup _{k \geq 0}\left|\phi_{k}\right|, \\
\ell_{0}\left(\mathbb{K}^{d}\right):=\left\{\phi=\left(\phi_{k}\right)_{k \geq 0}: \lim _{k \rightarrow \infty} \phi_{k}=0\right\}, & & \|\phi\|_{\ell_{0}}:=\sup _{k \geq 0}\left|\phi_{k}\right|, \\
\ell^{p}\left(\mathbb{K}^{d}\right):=\left\{\phi=\left(\phi_{k}\right)_{k \geq 0}: \sum_{k \geq 0}\left|\phi_{k}\right|^{p}<\infty\right\}, & \|\phi\|_{\ell^{p}}:=\sqrt[n]{\sum_{k \geq 0}\left|\phi_{k}\right|^{p}}
\end{array}
$$

with a real number $p \geq 1$. For Banach spaces possessing a Schauder basis, thus in particular $\ell_{0}\left(\mathbb{K}^{d}\right)$ and $\ell^{p}\left(\mathbb{K}^{d}\right)$, the quantity $\chi_{X}(B) \geq 0$ can be computed explicitly (cf., for instance, [ADL97, pp. 35ff] in connection with [BG80, p. 23]) as follows:

$$
\begin{aligned}
& \chi_{\ell_{0}}(B)=\lim _{n \rightarrow \infty} \sup _{x \in B} \sup _{k>n}\left|x_{k}\right| \quad \text { for all } B \in \mathcal{B}\left(\ell_{0}\left(\mathbb{K}^{d}\right)\right), \\
& \chi_{\ell^{p}}(B)=\lim _{n \rightarrow \infty} \sup _{x \in B} \sqrt[p]{\sum_{k>n}\left|x_{k}\right|^{p}} \quad \text { for all } B \in \mathcal{B}\left(\ell^{p}\left(\mathbb{K}^{d}\right)\right) .
\end{aligned}
$$

\section{Asymptotic BeHAVIOR OF RECURSIONS}

We consider the discrete implicit initial value problem (IVP for short)

$$
\begin{aligned}
x_{k+1} & =f_{k}\left(x_{k}, x_{k+1}\right), \\
x_{0} & =\xi
\end{aligned}
$$

for initial values $\xi \in \mathbb{K}^{d}$ and a right-hand side $f_{k}: \mathbb{K}^{d} \times \mathbb{K}^{d} \rightarrow \mathbb{K}^{d}, k \in \mathbb{N}_{0}$. For simplicity reasons we suppose that $f_{k}$ is globally defined on the whole space $\mathbb{K}^{d} \times \mathbb{K}^{d}$ and satisfies global assumptions (see below) throughout. This seemingly severe restriction has to be related to the goals of our approach: On the one hand, under global assumptions which are rarely satisfied in applications, we can deduce global convergence results. On the other hand, though, in stability theory one frequently encounters the situation where a small set (typically a neighborhood of 0 ) is forward invariant under the dynamics of (3.1). Then an appropriate modification of $f_{k}$ outside such an invariant set using retraction mappings allows us to deduce local convergence criteria from our global results; we demonstrate this standard procedure in Example 4.4 below.

In order to embed (3.1), (3.2) into our functional-analytical framework we introduce

- the linear embedding operator $\mathcal{E}: \mathbb{K}^{d} \rightarrow \ell\left(\mathbb{K}^{d}\right), \mathcal{E} \xi:=(\xi, 0,0, \ldots)$,

- the linear right shift operator $\mathcal{S}: \ell\left(\mathbb{K}^{d}\right) \rightarrow \ell\left(\mathbb{K}^{d}\right), \mathcal{S} \phi:=\left(0, \phi_{0}, \phi_{1}, \ldots\right)$,

- the substitution operator $\mathcal{F}_{f}: \ell\left(\mathbb{K}^{d}\right) \rightarrow \ell\left(\mathbb{K}^{d}\right), \mathcal{F}_{f}(\phi):=\left(f_{k}\left(\phi_{k}, \phi_{k+1}\right)\right)_{k>0}$,

- $\mathcal{G}_{f}: \ell\left(\mathbb{K}^{d}\right) \times \mathbb{K}^{d} \rightarrow \ell\left(\mathbb{K}^{d}\right)$, given by

$$
\mathcal{G}_{f}(\phi, \xi):=\mathcal{E} \xi+\mathcal{S F}_{f}(\phi) .
$$

The operator $\mathcal{G}_{f}$ depends linearly on the right-hand side $f_{k}$ and, if $f_{k}: \mathbb{K}^{d} \times \mathbb{K}^{d} \rightarrow$ $\mathbb{K}^{d}$ is a linear mapping, then $\mathcal{G}_{f}$ becomes affine linear. Our fundamental device to reformulate the IVP (3.1), (3.2) as an operator equation in $\ell\left(\mathbb{K}^{d}\right)$ is given in 
Theorem 3.1. Let $\xi \in \mathbb{K}^{d}$ and $\phi \in \ell\left(\mathbb{K}^{d}\right)$ be a sequence in $\mathbb{K}^{d}$. Then $\phi$ is a solution of the IVP (3.1), (3.2) if and only if $\phi$ solves the fixed point equation

$$
\phi=\mathcal{G}_{f}(\phi, \xi) \text {. }
$$

For an explicit recursion (3.1), a fixed-point of the mapping $\mathcal{G}_{f}(\cdot, \xi)$ is unique.

Proof. We refer to [EP07, Theorem 3.3] for the easy proof.

To get a flavor of our methodology, we give a first application of the interplay between Theorems 2.1 and 3.1. Its proof is based on the continuous embedding $\ell^{p}\left(\mathbb{K}^{d}\right) \hookrightarrow \ell^{q}\left(\mathbb{K}^{d}\right)$ for $p \leq q$ and can be adapted to other pairs of continuously embedded sequence spaces.

Proposition 3.2. Let $p \geq 1$ and $f_{k}: \mathbb{K}^{d} \times \mathbb{K}^{d} \rightarrow \mathbb{K}^{d}$ be continuous satisfying:

(i) there exist sequences $a \in \ell^{p}(\mathbb{R}), b, c \in \ell_{0}(\mathbb{R})$ and reals $\gamma \geq 1$ such that

$$
\left|f_{k}(x, y)\right| \leq a_{k}+\max \left\{b_{k}|x|^{\gamma}, c_{k}|y|^{\gamma}\right\} \quad \text { for all } k \in \mathbb{N}_{0}, x, y \in \mathbb{K}^{d},
$$

(ii) the inequality $|\xi|+\|a\|_{\ell^{p}}+\left(\|b\|_{\ell_{0}}+\|c\|_{\ell_{0}}\right) \rho^{\gamma} \leq \rho$ admits a solution $\rho_{0}>0$. Then there exists a solution $\phi \in \bar{B}_{\rho_{0}}\left(\ell^{p}\left(\mathbb{K}^{d}\right)\right)$ of the IVP (3.1), (3.2).

Proof. Let $\xi \in \mathbb{K}^{d}$ be fixed and define $q:=\gamma p \geq p$. Let us introduce the linear operator $\mathcal{A}: D(\mathcal{A}) \subseteq \ell^{q}\left(\mathbb{K}^{d}\right) \rightarrow \ell^{p}\left(\mathbb{K}^{d}\right), \mathcal{A} \phi:=\phi$ with $D(\mathcal{A}):=\ell^{p}\left(\mathbb{K}^{d}\right)$. Owing to the continuous embedding $\ell^{p}\left(\mathbb{K}^{d}\right) \hookrightarrow \ell^{q}\left(\mathbb{K}^{d}\right)$ we know that the inverse $\mathcal{A}^{-1}$ : $\ell^{p}\left(\mathbb{K}^{d}\right) \rightarrow \ell^{q}\left(\mathbb{K}^{d}\right)$ exists as a continuous mapping with norm 1 .

On the other hand, from our preliminaries in [EP07, Lemma 5.2, 5.3] we obtain that $\mathcal{G}_{f}(\cdot, \xi): \ell^{q}\left(\mathbb{K}^{d}\right) \rightarrow \ell^{p}\left(\mathbb{K}^{d}\right)$ is completely continuous with

$$
\left\|\mathcal{G}_{f}(\phi, \xi)\right\|_{\ell^{p}} \leq|\xi|+\|a\|_{\ell^{p}}+\left(\|b\|_{\ell_{0}}+\|c\|_{\ell_{0}}\right)\|\phi\|_{\ell^{q}}^{\gamma} \text { for all } \phi \in \ell^{q}\left(\mathbb{K}^{d}\right),
$$

and by Theorem 2.1 with $X=\ell^{q}\left(\mathbb{K}^{d}\right)$ and $Y=\ell^{p}\left(\mathbb{K}^{d}\right)$ there exists a $\phi \in \bar{B}_{\rho_{0}}\left(\ell^{p}\left(\mathbb{K}^{d}\right)\right)$ such that $\phi=\mathcal{A} \phi=\mathcal{G}_{f}(\phi, \xi)$. The assertion follows from Theorem 3.1 .

A crucial terminology for our investigations is the notion of admissibility, which in the linear case dates back to CS67. Thereto, the admissibility of subspaces $X, Y \subseteq \ell\left(\mathbb{K}^{d}\right)$ means that inputs $\psi \in Y$ of the form (1.2) into (3.1) produce unique outputs in $X$. To be more precise, we say that the difference equation (3.1) is $(X, Y)$-admissible if for each perturbation $\psi \in Y$ there exists a unique forward solution $\mathcal{J}(\psi) \in X$ of the IVP (1.2) and such that the admissibility map $\mathcal{J}: Y \rightarrow X$ satisfies $\operatorname{lip} \mathcal{J}<\infty$.

Theorem 3.3. Let $\xi \in \mathbb{K}^{d}, X, Y \subseteq \ell\left(\mathbb{K}^{d}\right)$ be normed sequence subspaces, suppose that (3.1) is $(X, Y)$-admissible with admissibility map $\mathcal{J}$ and

$$
x=f_{k}(0, x) \Leftrightarrow x=0 \quad \text { for all } k \in \mathbb{N}_{0} .
$$

If the mappings $g_{k}: \mathbb{K}^{d} \times \mathbb{K}^{d} \rightarrow \mathbb{K}^{d}$ satisfy

(i) there exist reals $\alpha, \beta, \gamma \geq 0$ such that

$$
\left\|\mathcal{G}_{g}(\phi, \xi)\right\|_{Y} \leq \alpha+\beta\|\phi\|_{X}^{\gamma} \quad \text { for all } \phi \in X,
$$

(ii) $\mathcal{F}_{g}: X \rightarrow Y$ is continuous and there exists a $\lambda \geq 0$ such that

$$
\chi_{Y}\left(\mathcal{F}_{g}(B)\right) \leq \lambda \chi_{X}(B) \quad \text { for all } B \in \mathcal{B}(X),
$$

(iii) $\lambda \operatorname{lip} \mathcal{J}<1$ and the inequality $\left(\alpha+\beta \rho^{\gamma}\right) \operatorname{lip} \mathcal{J} \leq \rho$ admits a solution $\rho_{0}>0$, 
then the IVP

$$
x_{k+1}=f_{k}\left(x_{k}, x_{k+1}\right)+g_{k}\left(x_{k}, x_{k+1}\right), \quad x_{0}=\xi
$$

possesses a solution in $\bar{B}_{\rho_{0}}(X)$.

Proof. Suppose $\xi \in \mathbb{K}^{d}$. Thanks to our basic Theorem 3.1 we know that every solution $\phi$ of the IVP (3.7) satisfies the fixed point equation $\phi=\mathcal{E} \xi+\mathcal{S F}_{f}(\phi)+$ $\mathcal{S F}_{g}(\phi)$ (cf. (3.3)) or equivalently the nonlinear equation

$$
\mathcal{A}(\phi)=\mathcal{G}_{g}(\phi, \xi)
$$

with the operator $\mathcal{A}: \ell\left(\mathbb{K}^{d}\right) \rightarrow \ell\left(\mathbb{K}^{d}\right), \mathcal{A}(\phi):=\phi-\mathcal{S} \mathcal{F}_{f}(\phi)$. In order to utilize Theorem 2.1 we choose $\psi \in Y$ and remark that $\mathcal{A}(\phi)=\psi$ has the explicit formulation

$$
\phi_{0}=\psi_{0}, \quad \phi_{k+1}-f_{k}\left(\phi_{k}, \phi_{k+1}\right)=\psi_{k+1} \quad \text { for all } k \in \mathbb{N}_{0} .
$$

Hence, due to the assumed $(X, Y)$-admissibility of (3.1) we know that there exists a unique sequence $\phi \in X$ such that $\mathcal{A}(\phi)=\psi$ holds, i.e., the admissibility map $\mathcal{J}$ : $Y \rightarrow X$ is the inverse of $\mathcal{A}$. In addition, from (3.5) we get $\mathcal{J}(0)=0$. The translation invariance of $\chi_{Y}$ implies $\chi_{Y}\left(\mathcal{G}_{g}(B, \xi)\right)=\chi_{Y}\left(\mathcal{F}_{g}(B)\right)$ and our assumptions yield

$$
\chi_{X}\left(\mathcal{A}^{-1}\left(\mathcal{G}_{g}(B, \xi)\right)\right) \leq \operatorname{lip} \mathcal{A}^{-1} \chi_{Y}\left(\mathcal{G}_{g}(B, \xi)\right)=\operatorname{lip} \mathcal{J} \chi_{Y}\left(\mathcal{F}_{g}(B)\right) \leq \lambda \operatorname{lip} \mathcal{J} \chi_{X}(B)
$$

for all $B \in \mathcal{B}(X)$. Consequently, $\mathcal{A}^{-1} \circ \mathcal{G}_{g}(\cdot, \xi)$ is a set-contraction. Then Theorem 2.1 guarantees that (3.8) has a solution in $\bar{B}_{\rho_{0}}(X)$, which by Theorem 3.1 is our claim.

\section{Admissibility and applications}

We continue this paper with three explicit applications of Theorem 3.3 A particularly interesting special case of (3.1) are linear difference equations of the form

$$
x_{k+1}=A_{k} x_{k}+B_{k} x_{k+1}
$$

with matrices $A_{k}, B_{k} \in \mathbb{K}^{d \times d}$ such that $I-B_{k}$ is invertible for every $k \in \mathbb{N}_{0}$. Then the transition operator $\Phi(k, n) \in \mathbb{K}^{d \times d}$ of (4.1) is given by

$$
\Phi(k, n):=\left\{\begin{array}{cl}
I & \text { for } k=n, \\
\left(I-B_{k-1}\right)^{-1} A_{k-1} \cdots\left(I-B_{n}\right)^{-1} A_{n} & \text { for } k>n .
\end{array}\right.
$$

We will see that admissibility properties for (4.1) can be derived using fairly classical operator-theoretical tools from, for example, $\operatorname{Mad} 70$, Wil84]. A different approach to the admissibility of linear difference equations can be found in CS67, NP97, Sas06 and related results under semi-linear perturbations are considered in Pin98.

Lemma $4.1\left(\left(\ell^{p}, \ell^{p}\right)\right.$-admissibility). Let $p, q>1$ be reals with $\frac{1}{p}+\frac{1}{q}=1$. If we have

$$
\begin{aligned}
& \nu_{1}:=\sup _{k \geq 0}|\Phi(k, 0)|+\sup _{k \geq 1} \sum_{n=1}^{k}\left|\Phi(k, n)\left(I-B_{n-1}\right)^{-1}\right|<\infty, \\
& \nu_{2}:=\max \left\{\sum_{k=0}^{\infty}|\Phi(k, 0)|, \sup _{n \geq 1} \sum_{k=n}^{\infty}\left|\Phi(k, n)\left(I-B_{n-1}\right)^{-1}\right|\right\}<\infty,
\end{aligned}
$$

then the linear difference equation (4.1) is $\left(\ell^{p}\left(\mathbb{K}^{d}\right), \ell^{p}\left(\mathbb{K}^{d}\right)\right)$-admissible with linear admissibility map $\mathcal{J}$ satisfying

$$
\operatorname{lip} \mathcal{J} \leq \sqrt[q]{\nu_{1}} \sqrt[p]{\nu_{2}}
$$


Example 4.2. Let $\theta<1$ be given. For constant matrices $A, B \in \mathbb{K}^{d \times d}$ the admissibility conditions (4.2) hold if the eigenvalues $\lambda \in \mathbb{C}$ of $(I-B)^{-1} A \in \mathbb{K}^{d \times d}$ satisfy $|\lambda| \leq \theta$ and such that eigenvalues with modulus $|\lambda|=\theta$ are semi-simple. Indeed, we have

$$
\nu_{1} \leq 1+\frac{1}{1-\theta}\left|(I-B)^{-1}\right|, \quad \nu_{2} \leq \frac{1}{1-\theta} \max \left\{1,\left|(I-B)^{-1}\right|\right\} .
$$

Proof of Lemma 4.1. Let $\psi \in \ell^{p}\left(\mathbb{K}^{d}\right)$. It is not difficult to see that the sequence

$$
\phi_{k}:=\Phi(k, 0) \psi_{0}+\sum_{n=0}^{k-1} \Phi(k, n+1)\left(I-B_{n}\right)^{-1} \psi_{n+1} \quad \text { for all } k \in \mathbb{N}_{0}
$$

is the unique forward solution of the perturbed linear difference equation

$$
x_{k+1}=A_{k} x_{k}+B_{k} x_{k+1}+\psi_{k+1}
$$

satisfying $\phi_{0}=\psi_{0}$. In order to deduce the inclusion $\phi \in \ell^{p}\left(\mathbb{K}^{d}\right)$, we interpret (4.4) formally as rows of a linear equation $\phi=\mathcal{J} \psi$, where the infinite matrix $\mathcal{J}$ is lower triangular. Adopting the well-definedness condition for matrix operators $\mathcal{J}$ from $\ell^{p}$ to $\ell^{p}$ given in $\left[\operatorname{Mad70}\right.$, p. 174, Theorem 9], we arrive at $\|\phi\|_{\ell^{p}} \leq \sqrt[q]{\nu_{1}} \sqrt[p]{\nu_{2}}\|\psi\|_{\ell^{p}}$. Thus, the linear mapping $\mathcal{J}(\psi)=\phi$ is our desired admissibility map satisfying (4.3).

Proposition 4.3. Let $\xi \in \mathbb{K}^{d}$ and $p, q>1$ be reals with $\frac{1}{p}+\frac{1}{q}=1$. Suppose the estimates (4.2) hold and that the continuous mappings $g_{k}: \mathbb{K}^{d} \times \mathbb{K}^{d} \rightarrow \mathbb{K}^{d}$ satisfy:

(i) there exist sequences $a \in \ell^{p}(\mathbb{R}), b, c \in \ell^{\infty}(\mathbb{R})$ such that

$$
\left|g_{k}(x, y)\right| \leq a_{k}+\max \left\{b_{k}|x|, c_{k}|y|\right\} \quad \text { for all } k \in \mathbb{N}_{0}, x, y \in \mathbb{K}^{d},
$$

(ii) $\sqrt[q]{\nu_{1}} \sqrt[p]{\nu_{2}}\left(\|b\|_{\ell^{\infty}}+\|c\|_{\ell^{\infty}}\right)<1$.

Then there exists a solution $\phi \in \bar{B}_{\rho_{0}}\left(\ell^{p}\left(\mathbb{K}^{d}\right)\right)$ of the semi-linear IVP

$$
x_{k+1}=A_{k} x_{k}+B_{k} x_{k+1}+g_{k}\left(x_{k}, x_{k+1}\right), \quad x_{0}=\xi,
$$

where we have

$$
\rho_{0}:=\frac{|\xi|+\sqrt[q]{\nu_{1}} \sqrt[p]{\nu_{2}}\|a\|_{\ell^{p}}}{1-\sqrt[q]{\nu_{1}} \sqrt[p]{\nu_{2}}\left(\|b\|_{\ell^{\infty}}+\|c\|_{\ell^{\infty}}\right)} .
$$

Proof. Let $\xi \in \mathbb{K}^{d}$ be given and successively check the assumptions of Theorem 3.3 with Banach spaces $X=\ell^{p}\left(\mathbb{K}^{d}\right), Y=\ell^{p}\left(\mathbb{K}^{d}\right)$. Above all, Lemma 4.1 guarantees that (4.1) is $\left(\ell^{p}\left(\mathbb{K}^{d}\right), \ell^{p}\left(\mathbb{K}^{d}\right)\right)$-admissible with an admissibility map $\mathcal{J}$ satisfying (4.3). From the invertibility of $I-B_{k}$ we obtain (3.5). Moreover, DG05, Theorems 1.1, 1.3] implies that $\mathcal{G}_{g}: \ell^{p}\left(\mathbb{K}^{d}\right) \times \mathbb{K}^{d} \rightarrow \ell^{p}\left(\mathbb{K}^{d}\right)$ is well-defined and continuous in the first argument. Due to [EP07, Lemma 5.2] we see that

$$
\left\|\mathcal{G}_{f}(\phi, \xi)\right\|_{\ell^{p}} \leq|\xi|+\|a\|_{\ell^{p}}+\left(\|b\|_{\ell^{\infty}}+\|c\|_{\ell^{\infty}}\right)\|\phi\|_{\ell^{p}} \quad \text { for all } \phi \in \ell^{p}\left(\mathbb{K}^{d}\right)
$$

and this yields (3.6) with $\alpha=|\xi|+\|a\|_{\ell^{p}}, \beta=\|b\|_{\ell^{\infty}}+\|c\|_{\ell^{\infty}}$ and $\gamma=1$. Now let $B \subseteq \ell^{p}\left(\mathbb{K}^{d}\right)$ be a bounded set and choose $\phi \in B$. Due to the translation invariance 
of the measure of noncompactness $\chi_{\ell^{p}}$ we can suppose $g_{k}(0,0) \equiv 0$ in the estimate

$$
\begin{aligned}
& \sqrt[p]{\sum_{k>n}\left|g_{k}\left(\phi_{k}, \phi_{k+1}\right)\right|^{p}} \leq \sqrt[p]{\sum_{k>n} \max \left\{b_{k}\left|\phi_{k}\right|, c_{k}\left|\phi_{k+1}\right|\right\}^{p}} \\
\leq & \sqrt[p]{\sum_{k>n}\left(b_{k}\left|\phi_{k}\right|+c_{k}\left|\phi_{k+1}\right|\right)^{p}} \leq\left(\|b\|_{\ell \infty}+\|c\|_{\ell \infty}\right) \sqrt[p]{\sum_{k>n}\left|\phi_{k}\right|^{p}} \\
\leq & \left(\|b\|_{\ell \infty}+\|c\|_{\ell \infty}\right) \sup _{\phi \in B} \sqrt{\sum_{k>n}\left|\phi_{k}\right|^{p}} \text { for all } n \in \mathbb{N}_{0} ;
\end{aligned}
$$

passing to the supremum over all $\phi \in B$ and taking the limit $n \rightarrow \infty$ leads to the inequality $\chi_{\ell^{p}}\left(\mathcal{F}_{g}(B)\right) \leq\left(\|b\|_{\ell^{\infty}}+\|c\|_{\ell^{\infty}}\right) \chi_{\ell^{p}}(B)$. Hence, our assumptions are sufficient for the hypotheses of Theorem 3.3

The following illustrative example demonstrates that Proposition 4.3, as well as our method in general, are easily applicable to implicit problems.

Example 4.4. Let us consider the nonautonomous implicit complex recursion

$$
z_{k+1}=\alpha z_{k}+\gamma_{k}\left|z_{k}\right|\left|z_{k+1}\right|+\delta_{k},
$$

where $\alpha \in \mathbb{C}$ with $|\alpha|<1,\left(\gamma_{k}\right)_{k \geq 0} \in \ell^{\infty}(\mathbb{C})$ and $\left(\delta_{k}\right)_{k \geq 0} \in \ell^{p}(\mathbb{C})$ for some $p>1$. We are using the radial retraction $R_{r}: \mathbb{C} \rightarrow \mathbb{C}, r>0$, given by

$$
R_{r}(z):=\left\{\begin{array}{l}
z, \quad|z| \leq r \\
\frac{r}{|z|} z, \quad|z|>r
\end{array}\right.
$$

to prepare the nonlinear equation (4.7) as follows:

$$
z_{k+1}=\alpha z_{k}+\gamma_{k}\left|R_{r}\left(z_{k}\right)\right|\left|R_{r}\left(z_{k+1}\right)\right|+\delta_{k} .
$$

Note that recursion (4.8) satisfies the assumptions of Proposition 4.3 with $A_{k}=\alpha$, $B_{k}=0, g_{k}(z, w)=\gamma_{k}\left|R_{r}(z)\right|\left|R_{r}(w)\right|+\delta_{k}$ and constants $\nu_{1} \leq \frac{2-|\alpha|}{1-|\alpha|}, \nu_{2} \leq \frac{1}{1-|\alpha|}$, $a_{k}=\left|\delta_{k}\right|, b_{k}=c_{k}=r\left|\gamma_{k}\right|$, provided $r>0$ is chosen so small that

$$
2 \sqrt[q]{\nu_{1}} \sqrt[p]{\nu_{2}} \sup _{k \geq 0}\left|\gamma_{k}\right| r<1 .
$$

Hence, referring to Proposition 4.3 there exists a solution $\phi \in \bar{B}_{\rho_{0}}\left(\ell^{p}(\mathbb{C})\right)$ of (4.8) starting in $\zeta \in \mathbb{C}$, which in particular satisfies

$$
\left|\phi_{k}\right| \leq \sqrt[p]{\rho_{0}}=\sqrt[p]{\frac{|\zeta|+\sqrt[q]{\nu_{1}} \sqrt[p]{\nu_{2}}\|a\|_{\ell^{p}}}{1-2 \sqrt[q]{\nu_{1}} \sqrt[p]{\nu_{2}}\|\gamma\|_{\ell^{\infty}} r}} \quad \text { for all } k \in \mathbb{N}_{0} .
$$

With sufficiently small $\ell^{p}$-norm of $a$ and initial values $\zeta$ close of 0 , we have $\left|\phi_{k}\right| \leq r$ for all $k \in \mathbb{N}_{0}$. Consequently, for such values of $\zeta$, the solution $\phi$ stays in the ball $\bar{B}_{r}(\mathbb{C})$ and due to the fact that (4.7) and (4.8) coincide on $\bar{B}_{r}(\mathbb{C})$, the sequence $\phi \in \bar{B}_{\rho_{0}}\left(\ell^{p}(\mathbb{C})\right)$ is also a solution of the original equation (4.7) starting in $\zeta$.

Lemma $4.5\left(\left(\ell_{0}, \ell_{0}\right)\right.$-admissibility $)$. If we have

$$
\begin{gathered}
\lim _{k \rightarrow \infty} \Phi(k, n)=0 \quad \text { for all } n \geq 0, \\
\nu:=\sup _{k \geq 0}|\Phi(k, 0)|+\sup _{k \geq 1} \sum_{n=1}^{k}\left|\Phi(k, n)\left(I-B_{n-1}\right)^{-1}\right|<\infty,
\end{gathered}
$$


then the linear difference equation (4.1) is $\left(\ell_{0}\left(\mathbb{K}^{d}\right), \ell_{0}\left(\mathbb{K}^{d}\right)\right)$-admissible with linear admissibility map $\mathcal{J}$ satisfying

$$
\operatorname{lip} \mathcal{J} \leq \nu .
$$

Example 4.6. Under the assumptions of Example 4.2 the admissibility conditions (4.9) are satisfied with $\nu \leq 1+\frac{1}{1-\theta}\left|(I-B)^{-1}\right|$.

Proof of Lemma 4.5. For a given inhomogeneity $\psi \in \ell_{0}\left(\mathbb{K}^{d}\right)$ we again define the uniquely determined forward solution $\phi$ of (4.5) satisfying $\phi_{0}=\psi_{0}$ by (4.4). Arguing as in the proof of Lemma 4.1 we deduce from [Mad70, p. 163, Theorem 1] that $\phi \in \ell_{0}\left(\mathbb{K}^{d}\right)$ with $\|\phi\|_{\ell_{0}} \leq \nu\|\psi\|_{\ell_{0}}$. Thus, the claim follows with admissibility map $\mathcal{J}(\psi):=\phi$.

Proposition 4.7. Let $\xi \in \mathbb{K}^{d}$. Suppose the relations (4.9) hold and that the mappings $g_{k}: \mathbb{K}^{d} \times \mathbb{K}^{d} \rightarrow \mathbb{K}^{d}$ satisfy:

(i) for each $\epsilon>0$ there exists a $\delta>0$ such that for every $k \in \mathbb{N}_{0}$ and $x, \bar{x}, y, \bar{y} \in$ $\mathbb{K}^{d}$ with $|x-\bar{x}|,|y-\bar{y}|<\delta$ one has

$$
\left|g_{k}(x, y)-g_{k}(\bar{x}, \bar{y})\right|<\epsilon,
$$

(ii) there exist sequences $a \in \ell_{0}(\mathbb{R})$ and $b, c \in \ell^{\infty}(\mathbb{R})$ such that

$$
\left|g_{k}(x, y)\right| \leq a_{k}+\max \left\{b_{k}|x|, c_{k}|y|\right\} \quad \text { for all } k \in \mathbb{N}_{0}, x, y \in \mathbb{K}^{d},
$$

(iii) $\nu \max \left\{\|b\|_{\ell^{\infty}},\|c\|_{\ell^{\infty}}\right\}<1$.

Then there exists a solution $\phi \in \bar{B}_{\rho_{0}}\left(\ell_{0}\left(\mathbb{K}^{d}\right)\right)$ of the IVP (4.6), where we have

$$
\rho_{0}:=\frac{|\xi|+\nu\|a\|_{\ell_{0}}}{1-\nu \max \left\{\|b\|_{\ell^{\infty}},\|c\|_{\ell^{\infty}}\right\}} .
$$

Proof. For given initial value $\xi \in \mathbb{K}^{d}$, let us check the assumptions of Theorem 3.3 with spaces $X=Y=\ell_{0}\left(\mathbb{K}^{d}\right)$. By Lemma 4.5 the linear difference equation (4.1) is $\left(\ell_{0}\left(\mathbb{K}^{d}\right), \ell_{0}\left(\mathbb{K}^{d}\right)\right)$-admissible and (4.10) holds. In addition, by [EP07, Lemma 5.5] the mapping $\mathcal{G}_{g}: \ell_{0}\left(\mathbb{K}^{d}\right) \times \mathbb{K}^{d} \rightarrow \ell_{0}\left(\mathbb{K}^{d}\right)$ is well-defined with

$$
\left\|\mathcal{G}_{g}(\phi, \xi)\right\|_{\ell_{0}} \leq|\xi|+\|a\|_{\ell_{0}}+\max \left\{\|b\|_{\ell^{\infty}},\|c\|_{\ell^{\infty}}\right\}\|\phi\|_{\ell_{0}} \quad \text { for all } \phi \in \ell_{0}\left(\mathbb{K}^{d}\right)
$$

and therefore (3.6) holds with $\alpha=|\xi|+\|a\|_{\ell_{0}}, \beta=\max \left\{\|b\|_{\ell^{\infty}},\|c\|_{\ell^{\infty}}\right\}$ and $\gamma=1$. We leave it to the interested reader to show that our assumption (i) implies the continuity of $\mathcal{F}_{g}: \ell_{0}\left(\mathbb{K}^{d}\right) \rightarrow \ell_{0}\left(\mathbb{K}^{d}\right)$. Let $B \subseteq \ell_{0}\left(\mathbb{K}^{d}\right)$ be a bounded set and pick $\phi \in B$. The translation invariance of $\chi_{\ell_{0}}$ allows us to suppose $g_{k}(0,0) \equiv 0$ in the estimate

$$
\sup _{k>n}\left|\mathcal{F}_{g}(\phi)_{k}\right| \leq \sup _{k>n} \max \left\{b_{k}\left|\phi_{k}\right|, c_{k}\left|\phi_{k+1}\right|\right\} \leq \max \left\{\|b\|_{\ell^{\infty}},\|c\|_{\ell^{\infty}}\right\} \sup _{\phi \in B} \sup _{k>n}\|\phi\|_{\ell_{0}}
$$

for all $n \in \mathbb{N}_{0}$. We take the least upper bound for $\phi \in B$ and pass to the limit $n \rightarrow \infty$ to arrive at $\chi_{\ell_{0}}\left(\mathcal{F}_{g}(B)\right) \leq \max \left\{\|b\|_{\ell^{\infty}},\|c\|_{\ell^{\infty}}\right\} \chi_{\ell_{0}}(B)$ for $B \in \mathcal{B}\left(\ell_{0}\left(\mathbb{K}^{d}\right)\right)$. Having this available, our assertion follows from Theorem 3.3.

Example 4.8. We consider a linear inhomogeneous difference equation

$$
x_{k+1}=\left(A+C_{k}\right) x_{k}+\left(B+D_{k}\right) x_{k+1}+d_{k}
$$

with matrices $A, B, C_{k}, D_{k} \in \mathbb{K}^{d \times d}$ such that $I-B$ is invertible for $k \in \mathbb{N}_{0}$ and $A, B$ satisfy the stability assumptions stated in Example 4.2 moreover, suppose 
$d \in \ell_{0}\left(\mathbb{K}^{d}\right)$. Then the above Proposition 4.7 can be applied with $g_{k}(x, y)=C_{k} x+$ $D_{k} y, a_{k}=\left|d_{k}\right|, b_{k}=2\left|C_{k}\right|, c_{k}=2\left|D_{k}\right|$ and $\nu \leq 1+\frac{1}{1-\theta}\left|(I-B)^{-1}\right|$, provided the perturbations fulfill

$$
2 \nu \sup _{k \geq 0} \max \left\{\left|C_{k}\right|,\left|D_{k}\right|\right\}<1 .
$$

Hence, by Proposition 4.7 for every initial value $\xi \in \mathbb{K}^{d}$ there exists a forward solution of (4.11) decaying to 0 . In particular, the linear system (4.11) is asymptotically stable.

Lemma $4.9\left(\left(\ell^{p}, \ell^{1}\right)\right.$-admissibility). Let $p \geq 1$ be a real number. If we have

$$
\nu:=\max \left\{\sum_{k=0}^{\infty}|\Phi(k, 0)|^{p}, \sup _{k \geq 1} \sum_{n=1}^{k}\left|\Phi(k, n)\left(I-B_{n-1}\right)^{-1}\right|^{p}\right\}<\infty,
$$

then the linear difference equation (4.1) is $\left(\ell^{p}\left(\mathbb{K}^{d}\right), \ell^{1}\left(\mathbb{K}^{d}\right)\right)$-admissible with linear admissibility map $\mathcal{J}$ satisfying

$$
\operatorname{lip} \mathcal{J} \leq \sqrt[p]{\nu} .
$$

Example 4.10. In the situation of Example 4.2 the admissibility conditions (4.12) hold with $\nu \leq \frac{1}{1-\theta^{p}} \max \left\{1,\left|(I-B)^{-1}\right|^{p}\right\}$.

Proof of Lemma 4.9, Let $\psi \in \ell^{1}\left(\mathbb{K}^{d}\right)$. Analogous to the proof of Lemma 4.1 we define the unique forward solution $\phi$ of (4.5) satisfying $\phi_{0}=\psi_{0}$ by (4.4). Then Mad70, p. 167, Theorem 5] yields $\phi \in \ell^{p}\left(\mathbb{K}^{d}\right)$ and we set $\mathcal{J}(\psi):=\phi$ as admissibility map.

Proposition 4.11. Let $\xi \in \mathbb{K}^{d}$ and $p \geq 1$ be a real number. Suppose the estimate (4.12) holds and that the continuous mappings $g_{k}: \mathbb{K}^{d} \times \mathbb{K}^{d} \rightarrow \mathbb{K}^{d}$ satisfy:

(i) there exist sequences $a, b, c \in \ell^{1}(\mathbb{R})$ such that

$$
\left|g_{k}(x, y)\right| \leq a_{k}+\max \left\{b_{k}|x|^{p}, c_{k}|y|^{p}\right\} \quad \text { for all } k \in \mathbb{N}_{0}, x, y \in \mathbb{K}^{d},
$$

(ii) the inequality $\sqrt[p]{\nu}\left[|\xi|+\|a\|_{\ell^{1}}+\left(\|b\|_{\ell^{1}}+\|c\|_{\ell^{1}}\right)\right] \rho^{p} \leq \rho$ admits a solution $\rho_{0}>0$.

Then there exists a solution $\phi \in \bar{B}_{\rho_{0}}\left(\ell_{0}\left(\mathbb{K}^{d}\right)\right)$ of the IVP (4.6).

Proof. For given initial value $\xi \in \mathbb{K}^{d}$ we verify the assumptions of Theorem 3.3 with sequence spaces $X=\ell^{p}\left(\mathbb{K}^{d}\right)$ and $Y=\ell^{1}\left(\mathbb{K}^{d}\right)$. Having Lemma 4.9 at hand, we find that the linear difference equation (4.1) is $\left(\ell^{p}\left(\mathbb{K}^{d}\right), \ell^{1}\left(\mathbb{K}^{d}\right)\right)$-admissible and (4.13) holds. In addition, the mapping $\mathcal{G}_{g}: \ell^{p}\left(\mathbb{K}^{d}\right) \times \mathbb{K}^{d} \rightarrow \ell^{1}\left(\mathbb{K}^{d}\right)$ is well-defined, since we have

$$
\left\|\mathcal{G}_{g}(\phi, \xi)\right\|_{\ell^{1}}=|\xi|+\sum_{k \geq 0}\left|g_{k}\left(\phi_{k}, \phi_{k+1}\right)\right| \leq|\xi|+\|a\|_{\ell^{1}}+\left(\|b\|_{\ell^{1}}+\|c\|_{\ell^{1}}\right)\|\phi\|_{\ell^{p}}^{p}
$$

for all $\phi \in \ell^{p}\left(\mathbb{K}^{d}\right)$ and thus (3.6) holds with $\alpha=|\xi|+\|a\|_{\ell^{1}}, \beta=\|b\|_{\ell^{1}}+\|c\|_{\ell^{1}}$, $\gamma=p$. It is not difficult to show that $\mathcal{F}_{g}: \ell^{p}\left(\mathbb{K}^{d}\right) \rightarrow \ell^{1}\left(\mathbb{K}^{d}\right)$ is continuous (we again refer to DG05, Theorems 1.1, 1.3]). Now let $B \subseteq \ell^{p}\left(\mathbb{K}^{d}\right)$ be bounded and choose $\phi \in B$. As above, the translation invariance of $\chi_{\ell^{1}}$ allows us to suppose $g_{k}(0,0) \equiv 0$ in the estimate

$\sum_{k>n}\left|\mathcal{F}_{g}(\phi)_{k}\right| \leq \sum_{k>n} \max \left\{b_{k}\left|\phi_{k}\right|^{p}, c_{k}\left|\phi_{k+1}\right|^{p}\right\} \leq \sum_{k>n} b_{k} \sup _{\phi \in B}\|\phi\|_{\ell^{p}}^{p}+\sum_{k>n} c_{k} \sup _{\phi \in B}\|\phi\|_{\ell^{p}}^{p}$ 
for all $n \geq 0$. We take the least upper bound over $\phi \in B$ and pass to the limit $n \rightarrow \infty$ to arrive at $\chi_{\ell^{1}}\left(\mathcal{F}_{g}(B)\right)=0$ for $B \in \mathcal{B}\left(\ell_{0}\left(\mathbb{K}^{d}\right)\right)$; i.e., the mapping $\mathcal{F}_{g}: \ell^{p}\left(\mathbb{K}^{d}\right) \rightarrow$ $\ell^{1}\left(\mathbb{K}^{d}\right)$ is completely continuous. Then our assertion follows from Theorem 3.3 .

\section{ACKNOWLEDGMENT}

The author would like to thank the referee for insightful remarks improving the accessibility and quality of the paper.

\section{REFERENCES}

[ADL97] J. M. Ayerbe Toledano, T. Domínguez Benavides, and G. López Acedo, Measures of noncompactness in metric fixed point theory, Operator Theory: Advances and Applications 99, Birkhäuser Verlag, Basel, 1997. MR.1483889 (99e:47070)

[Aga00] R. P. Agarwal, Difference equations and inequalities, 2nd ed., Monogr. Textbooks Pure Appl. Math. 228, Marcel Dekker, New York, 2000. MR1740241 (2001f:39001)

[AZ90] J. Appell and P. P. Zabrejko, Nonlinear superposition operators, Cambridge University Press, Cambridge, 1990. MR.1066204 (91k:47168)

[BG80] J. Banaś and K. Goebel, Measures of noncompactness in Banach spaces, Lecture Notes in Pure and Applied Mathematics 60, Marcel Dekker, New York, 1980. MR.591679 (82f:47066)

[CS67] C. V. Coffman and J. J. Schäffer, Dichotomies for linear difference equations, Math. Ann. 172 (1967), 139-166. MR0214946 (35:5791)

[Dar55] G. Darbo, Punti uniti in trasformazioni a codominio non compatto, Rend. Sem. Mat. Univ. Padova 24 (1955), 84-92. MR0070164 (16:1140f)

[DG05] F. Dal and G. Sh. Guseinov, Properties of discrete composition operators, J. Difference Equ. Appl. 11 (2005), no. 1, 21-27. MR2112803 (2005g:47119)

[EP07] K. Ey and C. Pötzsche, Asymptotic behavior of recursions via fixed point theory, J. Math. Anal. Appl. 337 (2008), no. 2, 1125-1141. MR.2386362 (2009b:39015)

[Gor71] S. P. Gordon, Stability and summability of solutions of difference equations, Math. Syst. Theory 5 (1971), 56-65. MR0303141 (46:2279)

[Mad70] I. J. Maddox, Elements of Functional Analysis, Cambridge University Press, LondonNew York, 1970. MR0390692 (52:11515)

[NP97] R. Naulin and M. Pinto, Stability of discrete dichotomies for linear difference systems, J. Difference Equ. Appl. 3 (1997), no. 2, 101-123. MR1467605 (98g:39007)

[Pin98] M. Pinto, Weighted convergent and bounded solutions of difference systems, Comput. Math. Appl. 36 (1998), no. 10-12, 391-400. MR.1666156 (99j:39009)

[PS05] E. N. Petropoulou and P. D. Siafarikas, Existence of complex $\ell_{2}$ solutions of linear delay systems of difference equations, J. Difference Equ. Appl. 11 (2005), no. 1, 49-62. MR2112805 (2005h:39027)

[Sad67] B. N. Sadovskiǐ, On a fixed-point principle, Funct. Anal. Appl. 1 (1967), no. 2, 151-153. MR.0211302 (35:2184)

[Sas06] B. Sasu, Uniform dichotomy and exponential dichotomy of evolution families on the half-line, J. Math. Anal. Appl. 323 (2006), 1465-1478. MR.2260196 (2007j:34078)

[Wil84] A. Wilansky, Summability through functional analysis, North-Holland Mathematics Studies 85, North-Holland, Amsterdam, 1984. MR738632 (85d:40006)

Technische Universität München, Zentrum Mathematik, Boltzmannstrasse 3, D-85748 Garching, Germany

E-mail address: christian.poetzsche@ma.tum.de 